\title{
Cardiovascular safety of strontium ranelate: real-life assessment in clinical practice
}

\author{
A.-F. Donneau • J.-Y. Reginster
}

Received: 14 November 2013 / Accepted: 15 November 2013/Published online: 10 December 2013

(C) International Osteoporosis Foundation and National Osteoporosis Foundation 2013

The balance between the benefits and the risks of any medical treatment, action for prevention, or diagnostic procedure lies at the heart of any clinical decision. In line with this, the European Medicines Agency (EMA) recently set up a series of Good Pharmacovigilance Practices to reinforce procedures for surveillance and reporting of adverse events with authorised medical products [1]. These new regulations are currently being applied throughout all EU member states. In this context, the safety of all centrally registered drugs is closely monitored by the EMA through a new committee, the Pharmacovigilance Risk Assessment Committee (PRAC), which was launched in October 2012. The procedures include regular submission of periodic safety update reports (PSURs).

Naturally, treatments in osteoporosis are no exception to these regulations. In November 2012, the PSUR for strontium ranelate, which encompassed a number of new randomised clinical trials, included an updated assessment of the overall safety of the treatment and was submitted to the PRAC in accordance with the regulatory schedule. The overall safety analyses showed an increased cardiovascular risk in patients treated with strontium ranelate [2]. This ongoing process has led to a label change, and, in order to mitigate the cardiovascular risk, strontium ranelate is now contraindicated in patients with a history of cardiovascular disease, i.e. in patients with a history of ischaemic heart disease, peripheral artery disease, and/or cerebrovascular disease and in those with

A.-F. Donneau

Medical Biostatistics Unit, Department of Public Health Sciences,

University of Liège, Liege, Belgium

J.-Y. Reginster $(\bowtie)$

Department of Public Health and Health Economics,

University of Liège, 4020 Liege, Belgium

e-mail: jyreginster@ulg.ac.be uncontrolled hypertension. As a precaution, patients should now be evaluated for cardiovascular risk before starting treatment with strontium ranelate and at regular intervals during treatment.

In the light of these procedures, the results of two new studies that recently became available are published together in this issue of Osteoporosis International [3, 4]. Both constitute retrospective observational studies conducted in databases of electronic healthcare records and were set up to analyse the cardiovascular risk associated with the prescription of strontium ranelate in real-life clinical practice in the UK and in Denmark.

The two studies have contrasting sources of data and study design. The study presented by Cooper et al. [3] is a nested case-control study that combines longitudinal primary care data from the UK (Clinical Practice Research Datalink) with external National Health Service-linked datasets that provide information on the cause of death and hospitalisation. Abrahamsen et al. [4] report a traditional cohort study in the Danish National Prescription Database, which links data between national registries for dispensed prescriptions, hospitalisations, and causes of death for fatalities in Denmark.

The results of the studies are consistent on three points. First, observational data do not indicate that the use of strontium ranelate was associated with a significant increase in myocardial infarction. Cooper et al. compared the risk of ischaemic cardiac events in postmenopausal osteoporotic women who were currently receiving treatment with strontium ranelate — or had received it in the past - with the risk in those who had never received strontium ranelate [3]. Current use or past use of strontium ranelate was not associated with any significant increase in the risk for three cardiovascular events: first myocardial infarction, hospitalisation with myocardial infarction, or cardiovascular death. In their study, Abrahamsen calculated the incidence of myocardial infarction in men and postmenopausal women [4] and reported that the 
risk for myocardial infarction was not significantly elevated, though they did find a very borderline result for stroke and cardiovascular death and a significant increase in risk for allcause mortality.

Second, both studies highlighted substantial differences in patient profile of users of strontium ranelate compared with users of other osteoporosis treatments. Indeed, it appears that strontium ranelate patients are generally older, and - as would be expected for an older population - they have more severe osteoporosis and a longer duration of disease. They also have more co-morbidities, notably those related to elevated cardiovascular risk, such as cardiac failure $(22 \%$ in the Danish study), peripheral vascular disease (6\%), and cerebrovascular disease $(11 \%)$, with a combined prevalence of ischaemic heart disease, peripheral vascular disease, and cerebrovascular disease of $19 \%$ in women and $30 \%$ in men. The cases of ischaemic cardiac events in the UK study were also at substantially higher risk compared with the controls, with higher rates of history of hospitalisation for myocardial infarction (12 versus $4 \%$ ), ischaemic heart disease (71 versus $24 \%$ ), peripheral artery disease (18 versus $7 \%$ ), and cerebrovascular disease ( 23 versus $15 \%$ ). This is a significant finding for clinical practice: the majority of cases of myocardial infarction occurred in patients who would not be treated with the agent according to the new contraindications for strontium ranelate.

Third, while each study has its merits and limitations - and both are highly robust - neither study managed to properly address the major challenge of the potential channelling bias by which more fragile or severe patients being switched to strontium ranelate. Clearly, further research is warranted with appropriate handling of the remaining bias for a more complete evaluation of risk.

All osteoporosis treatments have their own inherent benefits and risks, and a clear-cut assessment of the benefit/risk ratio is important when they are to be used long term [5-7]. The role of the clinician is to select the best treatment for the patient's profile and individual therapeutic objective, which should remain the prevention of osteoporotic fracture [8]. By strictly applying the new contraindications for strontium ranelate, we can hope to achieve our primary goal of treating disease, preventing osteoporotic fracture, while markedly reducing the risk for side effects.

\section{Conflict of interest}

Name: Jean-Yves Reginster on behalf of the Department of Public Health, Epidemiology and Health Economics of the University of Liège, Liège, Belgium

Consulting fees or paid advisory boards: Servier, Novartis, Negma, Lilly, Wyeth, Amgen, GlaxoSmithKline, Roche, Merckle, NycomedTakeda, NPS, IBSA-Genevrier, Theramex, UCB, Asahi Kasei, Endocyte

Lecture fees when speaking at the invitation of a commercial sponsor: Merck Sharp and Dohme, Lilly, Rottapharm, IBSA, Genevrier, Novartis, Servier, Roche, GlaxoSmithKline, Merckle, Teijin, Teva, Analis, Theramex, Nycomed, NovoNordisk, Ebewee Pharma, Zodiac, Danone, Will Pharma, Amgen

Grant support from Industry: Bristol Myers Squibb, Merck Sharp \& Dohme, Rottapharm, Teva, Roche, Amgen, Lilly, Novartis, GlaxoSmithKline, Servier, Pfizer, Theramex, Danone, Organon, Therabel, Boehringer, Chiltern, Galapagos

Anne-Françoise Donneau has no competing interests.

\section{References}

1. European Medicines Agency (2012) Good pharmacovigilance practices. Available at: www.ema.europa.eu. Accessed 4 November 2013

2. European Medicines Agency (2013) PSUR assessment report for strontium ranelate. Available at: www.ema.europa.eu. Accessed 4 November 2013

3. Cooper C, Fox KM, Borer JS (2013) Ischaemic cardiac events and use of strontium ranelate in postmenopausal osteoporosis: a nested case-control study in the CPRD. Osteoporos Int. doi:10.1007/s00198-013-2582-4

4. Abrahamsen B, Grove EL, Vestergaard P (2013) Nationwide registrybased analysis of cardiovascular risk factors and adverse outcomes in patients treated with stronium ranelate. Osteoporos Int. doi:10.1007/ s00198-013-2469-4

5. Cooper C, Reginster JY, Cortet B et al (2012) Long-term treatment of osteoporosis in postmenopausal women: a review from the European Society for Clinical and Economic Aspects of Osteoporosis and Osteoarthritis (ESCEO) and the International Osteoporosis Foundation (IOF). Curr Med Res Opin 28:475-491

6. Rizzoli R, Reginster JY, Boonen S et al (2011) Adverse reactions and drug-drug interactions in the management of women with postmenopausal osteoporosis. Calcif Tissue Int 89:91-104

7. Rizzoli R, Reginster JY (2011) Adverse drug reactions to osteoporosis treatments. Expert Rev Clin Pharmacol 4:593-604

8. Kanis JA, McCloskey EV, Johansson H et al (2013) European guidance for the diagnosis and management of osteoporosis in postmenopausal women. Osteoporos Int 24:23-57 